\title{
Evolución del consumo de fármacos antiparkinsonianos en España
}

\author{
E. Montané ${ }^{\mathrm{a}}$, A. Vallano ${ }^{\mathrm{a}, \mathrm{b}, \mathrm{c}}$, J.M. Castel ${ }^{\mathrm{b}, \mathrm{c}}$
}

THE EVOLUTION OF USE OF ANTI-PARKINSON DRUGS IN SPAIN

\begin{abstract}
Summary. Introduction. In recent years new anti-Parkinson drugs have been marketed and there has been controversy over the safety of some drugs. Objective. To analyze the evolution of the consumption of anti-Parkinson drugs and the effect of the newer drugs. Patients and methods. A study of the consumption of anti-Parkinson drugs (1989-1998). Data were obtained from the ECOM database of the Ministry of Health and TEMPUS of the National Statistics Institute. The drugs were classified using the Anatomo-Therapeutic-Clinical Classification (ATC). Consumption was expressed in defined daily dosage (DDD) and the costs in euros (n). The drugs marketed since 1990 were classified as new drugs and the others as classical drugs. Results. The total consumption of drugs increased from 1.92 DDD/1,000 inhabitants/day in 1989 to 3.64 DDD/1,000 inhabitants/day in 1998. The drugs showing the greatest increase were selegiline, pergolide and levodopa. The total pharmaceutical expenses tripled. There was a smaller increase in the consumption of new drugs (1.2\% of the total in 1991 and $6.6 \%$ in 1998) than in their costs $(6.7 \%$ of the total in 1991 and $38.8 \%$ in 1998). The cost per DDD of the new drugs increased five times (1989: 2.55n and 1998: 13.59n) and that of the classical drugs was similar (1989:0.54n and 1998: 0.62n). Conclusions. The total consumption of anti-Parkinson drugs has progressively increased. The consumption of selegiline has also increased in spite of controversy over its safety. The new drugs have a major economic effect. [REV NEUROL 2002; 34: 612-7]
\end{abstract}

Key words. Antiparkinson agents. Costs and costs analysis. Drug costs. Drug therapy. Drug utilization. Parkinson disease.

\section{INTRODUCCIÓN}

La enfermedad de Parkinson (EP) es una alteración neurodegenerativa de causa desconocida, y de curso crónico, progresivo e irreversible [1-4]. Actualmente, es la segunda enfermedad neurodegenerativa más frecuente [4,5], después de la enfermedad de Alzheimer, y tiene una elevada repercusión sanitaria, porque incrementa la mortalidad, disminuye la calidad de vida de los pacientes y aumenta la necesidad de recursos sanitarios y económicos [4].

En los próximos años se prevé un aumento de la incidencia de la EP, como consecuencia del progresivo envejecimiento de la población $[1,6]$. El tratamiento de la EP es básicamente sintomático y tiene como objetivo mejorar los síntomas y signos de la enfermedad [1-3]. La levodopa es el fármaco antiparkinsoniano de referencia; sin embargo, en los últimos años han aparecido nuevos fármacos, como los agonistas dopaminérgicos, inhibidores de la monoaminoxidasa B (IMAO-B) y de la catecolo-metiltransferasa (COMT), con la finalidad de prevenir, suprimir o mejorar las complicaciones a largo plazo de la enfermedad, sobre todo las discinesias y fluctuaciones motoras [1-3]. Sin embargo, existe una controversia en relación con la seguridad de alguno de estos nuevos fármacos, como, por ejemplo, la selegilina [7] y la tolcapona [8,9].

Diversos estudios han analizado el consumo de fármacos antiparkinsonianos en España, pero se han limitado al ámbito geográfico de una comunidad autónoma; los de ámbito nacional, realizados hace más de una década, incluyeron un período reducido y se han limitado a evaluar exclusivamente el uso de

Recibido: 24.10.01. Aceptadotras revisión externa sinmodificaciones: 12.01.02.

${ }^{a}$ Servicio de Farmacología Clínica. Hospital Universitario Vall d'Hebron. ${ }^{b}$ Universitat Autònoma de Barcelona. ${ }^{c}$ Fundació Institut Català de Farmacologia. Barcelona, España.

Correspondencia: Dr. Antonio Vallano Ferraz. Servicio de Farmacología Clínica. Hospital Universitario Vall d'Hebron. Passeig de la Vall d'Hebron, 119-129. E-08035 Barcelona. Fax: +34 934894109.E-mail: tv@icf.uab.es

C 2002, REVISTA DE NEUROLOGÍA levodopa [10-15]. Además, en los últimos años se han comercializado nuevos fármacos antiparkinsonianos de los que no existen datos sobre su impacto en el consumo total de fármacos antiparkinsonianos. Por este motivo, el objetivo de nuestro estudio ha sido analizar la evolución del consumo de fármacos antiparkinsonianos en España durante un período de 10 años y el impacto de los nuevos fármacos comercializados. Un objetivo secundario del estudio fue evaluar el impacto que ha tenido sobre el consumo de selegilina la polémica relacionada con su seguridad.

\section{MATERIAL Y MÉTODOS}

Hemos diseñado un estudio sobre el consumo de fármacos antiparkinsonianos en España durante un período de 10 años, desde 1989 hasta 1998. Los datos de consumo de fármacos se obtuvieron de la base de datos ECOM, del Ministerio de Sanidad y Consumo, que contiene los datos -en número de envases e importe económico en pesetas-de todas las prescripciones de medicamentos dispensadas mediante recetas realizadas a cargo del Servicio Nacional de la Salud. Las cifras demográficas anuales de la población española se obtuvieron de la base de datos TEMPUS, del Instituto Nacional de Estadística. Se recogió información de las siguientes variables, mediante un cuaderno de recogida de datos estructurado: año, grupos farmacológicos antiparkinsonianos, tipo de fármacos antiparkinsonianos, unidades cuantitativas de consumo e importe económico del consumo de cada fármaco.

Los fármacos antiparkinsonianos se clasificaron mediante la Clasificación Anatómica-Terapéutica-Química (ATC) [16]. Se seleccionaron todas las especialidades farmacéuticas que pertenecían al grupo de fármacos antiparkinsonianos (N04), y que se comercializaron en España durante el período de estudio. Los medicamentos se clasificaron en dos grandes grupos: agentes anticolinérgicos (N04A) y agentes dopaminérgicos (N04B). La clasificación ATC ordena el grupo de los agentes dopaminérgicos en cinco subgrupos terapéuticos: dopa y derivados dopaminérgicos (NO4B A), derivados del adamantane (N04B B), agonistas dopaminérgicos (N04B C), inhibidores de la monoaminoxidasa tipo B (N04B D) y otros agentes dopaminérgicos (N04B X). Los fármacos comercializados desde 1990 (lisurida, pergolida, cabergolina, ropinirol y tolcapona) se clasificaron como nuevos fármacos antiparkinsonianos - pramipexol y entacapona todavía no se comercializaban durante el período de estudio-, y el resto de fármacos, es decir, los comercializados antes de este año, se clasificaron como fármacos 
clásicos. El consumo de fármacos se expresó en las dosis diarias definidas (DDD, año 1998) de los diferentes fármacos antiparkinsonianos, establecido por el Centro Colaborador de la OMS para Metodología en Estadísticas sobre Medicamentos. La tabla I indica la DDD de los diferentes fármacos antiparkinsonianos. El número de DDD consumidas se expresó por $1.000 \mathrm{o}$ por 100.000 habitantes y por día. La variación del consumo durante el período de estudio se calculó mediante el cociente entre la diferencia del consumo de 1998 y de 1989 y el consumo en 1989 [(consumo 1998 - consumo 1989/consumo 1989) $\times 100$ ]. El gasto farmacéutico y el coste por DDD se expresaron en euros (n). El coste por DDD de los fármacos clásicos y de los nuevos fármacos se calculó mediante el cociente entre el gasto total de los fármacos y el número de DDD de los fármacos. El análisis estadístico de los datos se llevó a cabo mediante el paquete estadístico SPSS Windows versión 9. Se realizó una estadística descriptiva: frecuencias, media y desviación estándar (DE) de las variables analizadas.

\section{RESULTADOS}

La media (DE) del consumo anual durante el período de estudio fue de $2,82(0,6) \mathrm{DDD} / 1.000$ habitantes/día. La figura 1 muestra la evolución anual del consumo total de fármacos antiparkinsonianos (N04) y de los dos principales grupos terapéuticos (N04A y N04B). Se observa que el aumento del consumo total se debió al incremento del consumo de los agentes dopaminérgicos (N04B), que progresó durante todo el período de estudio (desde un 55\% del consumo total en 1989 hasta un 75\% del consumo en 1998). En la tabla II se aprecia la evolución del consumo de los diferentes fármacos del grupo de los agentes anticolinérgicos. El fármaco anticolinérgico de mayor consumo fue biperideno. En la tabla III se presenta la evolución del consumo de los fármacos dopaminérgicos. Levodopa y selegilina fueron los fármacos de mayor consumo durante el período de estudio, pero los fármacos con un mayor incremento del consumo fueron ropirinol, selegilina y pergolida. En la figura 2 se muestra la evolución del consumo anual de selegilina en relación con la publicación de los resultados del estudio del grupo PDRGUK (Parkinson's Disease Research Group of the United Kingdom) [7].

El coste total también se incrementó progresivamente durante el período del estudio. El gasto total del consumo de fármacos antiparkinsonianos fue de $14.364 .189 \mathrm{n}$ ( 2.390 millones de ptas.) en el año 1989 y de $49.463 .296 \mathrm{n}$ (8.230 millones de ptas.) en el año 1998. Este crecimiento del gasto se debió, sobre todo, al aumento del gasto de los agentes dopaminérgicos (N04B), que se incrementó desde $11.599 .533 \mathrm{n}$ (1.930 millones de ptas.) en 1989 hasta 46.217.830n (7.690 millones de ptas.) en 1998. La media (DE) anual del coste de la DDD de los fármacos en el período de estudio fue $0,68(0,14) \mathrm{n}$ [112 $(22,7)$ ptas.]. El coste de la DDD se incrementó desde $0,51 \mathrm{n}$ ( 85 ptas.) en 1989 hasta 0,95 n (158 ptas.) en 1998.

El consumo de los nuevos fármacos aumentó de forma paulatina hasta 1997, pero en 1998 se observó un crecimiento súbito (más del 6\%), debido fundamentalmente al destacado consumo de tolcapona (Tabla IV). El gasto económico de los nuevos fármacos se incrementó desde 30.050n (5 millones de ptas.) hasta 19.232.387n (3.200 millones de ptas.). En el año 1998, el importe económico de los nuevos fármacos fue un $39 \%$ del gasto total de fármacos (Tabla V). El coste medio de la DDD de los nuevos fármacos se multiplicó por 7,3 desde 1990 (1,86n [310 ptas.]) hasta 1998 (13,59n [2.262 ptas.]). En cambio, el aumento del coste medio de la DDD de los fármacos clásicos sólo se multiplicó por 1,2 (Tabla V).

\section{DISCUSIÓN}

El consumo total de fármacos antiparkinsonianos ha aumentado en España durante la última década. Los resultados de varios estudios de ámbito nacional y en diferentes períodos muestran un progresivo crecimiento del consumo de los fármacos antiparkinsonianos [10-15]. Este incremento del consumo puede relacionarse con un aumento de la incidencia de la EP y el progresivo envejecimiento de la población; y también puede indicar un aumento del número de los pacientes que se tratan -con la EP o en otras indicaciones- y del número de fármacos utilizados en el tratamiento. Sin embargo, se ha señalado que el
Tabla I. Dosis diarias definidas (DDD), fecha de comercialización e indicación autorizada de los diferentes fármacos antiparkinsonianos.

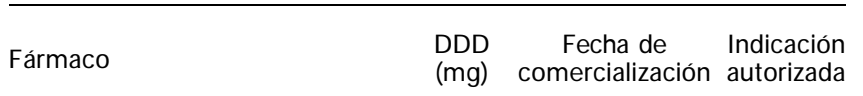

Agentes anticolinérgicos

\begin{tabular}{lccl}
\hline Aminas terciarias & & & \\
\hline Trihexifenidilo & 10 & $5 / 1967$ & EP y SE \\
\hline Biperideno & 10 & $7 / 1973$ & EP y SE \\
\hline Metixeno & 40 & $2 / 1967$ & O $^{\text {a }}$ \\
\hline Prociclidina & 25 & $2 / 1967$ & EP y SE \\
\hline Bornaprina & 9 & $9 / 1982^{\text {d }}$ & EP y SE
\end{tabular}

Agentes dopaminérgicos

Dopa y derivados de dopa

\begin{tabular}{lrll}
\hline Levodopa & 3.600 & $11 / 1973$ & EP \\
\hline Levodopa + benseracida & 600 & & EP \\
\hline Levodopa + carbidopa & 600 & & EP \\
\hline
\end{tabular}

Derivados de adamantane

\begin{tabular}{llll}
\hline Amantadina & 200 & $1 / 1968$ & $\mathrm{O}^{\mathrm{b}}$ \\
\hline
\end{tabular}

Agonistas dopaminérgicos

\begin{tabular}{llll}
\hline Bromocriptina & 40 & $7 / 1979$ & EP \\
\hline Pergolida & 3 & $1 / 1991$ & EP \\
\hline Ropinirol & 6 & $8 / 1997$ & EP \\
\hline Pramipexol & 3,5 & $12 / 1998$ & EP \\
\hline Cabergolina & 4 & $1 / 1996$ & O $^{c}$ \\
\hline Lisurida & 1,5 & $5 / 1990$ & EP \\
\hline IM AO tipo B & & & \\
\hline Selegilina & 5 & $1 / 1989$ & EP \\
\hline
\end{tabular}

Otros agentes dopaminérgicos

\begin{tabular}{lrcl}
\hline Tolcapona & 450 & $12 / 1997^{\mathrm{e}}$ & EP \\
\hline Entacapona & 1000 & $1 / 1999$ & EP \\
\hline
\end{tabular}

EP: enfermedad de Parkinson; SE: síntomas extrapiramidales; ${ }^{a}$ Otras indicaciones: meteorismo, insuficiencia hepática, pancreática y digestiva; ${ }^{\mathrm{b}}$ Otras indicaciones: profilaxis y tratamiento de la gripe $A ;{ }^{c}$ Otras indicaciones: hiperprolactinemia y supresión e inhibición de la lactancia. ${ }^{\mathrm{d}}$ Retirada del mercado en mayo de 1996; ' Retirada del mercado en noviembre de 1998. La DDD de bomaprina no la define el Centro Colaborador de la OMS para Metodología en Estadísticas sobre Medicamentos de 1999; se definió una DDD de 9 mg.

consumo de fármacos antiparkinsonianos en España todavía es inferior al de otros países, como los nórdicos [14,17-21]. Estas diferencias internacionales del consumo quizás se deben a diferencias diagnósticas y terapéuticas, porque algunos estudios indican que todavía hay pacientes con EP no diagnosticados $[22,23]$, aunque también pueden reflejar la existencia de variaciones internacionales sociodemográficas, genéticas y medioambientales.

La levodopa es el fármaco antiparkinsoniano de elección y 
Tabla II. Evolución anual del consumo de anticolinérgicos (N04A) (expresado en DDD/100.000 habitantes/día).

\begin{tabular}{lrrrrrrrrrrr}
\hline Fámmacos N04A A X (DE) anual & 1989 & 1990 & 1991 & 1992 & 1993 & 1994 & 1995 & 1996 & 1997 & 1998 & $\%$ var. \\
\hline Biperideno 65,7 (7,9) & 53,2 & 56,7 & 61,1 & 63,8 & 64,6 & 65,7 & 67,1 & 80,3 & 72,5 & 72,1 & $+35,5$ \\
\hline Bomaprina 2,2 (1,5) & 4,1 & 3,8 & 3,4 & 3,1 & 2,6 & 2,3 & 1,9 & 0,7 & $<0,1$ & $<0,1$ & $-99,5$ \\
\hline Metixeno 0,5 (0,3) & 0,9 & 0,8 & 0,8 & 0,7 & 0,7 & 0,6 & 0,6 & 0,4 & $<0,1$ & $<0,1$ & $-99,9$ \\
\hline Prociclidina 0,5 (0,07) & 0,6 & 0,5 & 0,5 & 0,5 & 0,4 & 0,4 & 0,4 & 0,4 & 0,5 & 0,5 & $-16,6$ \\
\hline Trihexifenidilo 23,9 (2,5) & 26,2 & 26,2 & 26,4 & 26,1 & 24,7 & 23,9 & 23,1 & 22,5 & 21,4 & 18,6 & $-29,0$ \\
\hline
\end{tabular}

$X(D E)$ anual: media de consumo anual durante todo el período de estudio; \% var.: porcentaje de variación [(consumo 1998 - consumo 1989)/consumo 1989]× 100.

Tabla III. Evolución anual del consumo de fármacos dopaminérgicos (N04B) (expresado en DDD/100.000 habitantes/día).

\begin{tabular}{lrrrrrrrrrrr}
\hline Fámacos N04B X (DE) anual & 1989 & 1990 & 1991 & 1992 & 1993 & 1994 & 1995 & 1996 & 1997 & 1998 & $\%$ var. \\
\hline N04B A Levodopa 113 (20) & 89,2 & 92,5 & 98,8 & 102,4 & 107,7 & 112,5 & 118,7 & 128,4 & 136,8 & 148,8 & $+66,8$ \\
\hline N04B B Amantadina 3,1 (0,7) & 4,0 & 3,9 & 3,7 & 3,5 & 3,3 & 3,2 & 2,5 & 2,1 & 3,0 & 3,0 & -25 \\
\hline N04B C Bromocriptina 7,5 (0,5) & 7,2 & 7,5 & 7,7 & 7,9 & 7,9 & 7,8 & 7,8 & 8,0 & 7,2 & 6,3 & $-12,5$ \\
\hline Pergolida 6,3 (3,4) & & 1,1 & 3,4 & 4,5 & 5,5 & 6,6 & 8,3 & 10,1 & 11,2 & $+918^{\text {a }}$ \\
\hline Ropinirol 2,1 (2,6) & & & & & & & & 0,3 & 4 & $1233^{\text {b }}$ \\
\hline Cabergolina 0,1 (0,1) & & & & & & & $<, 1$ & 0,1 & 0,2 & $+100^{\text {b }}$ \\
\hline Lisurida 3,1 (1,4) & - & 0,1 & 2,4 & 3,7 & 4,3 & 4,5 & 4,2 & 3,8 & 3,2 & 2,5 & $+4^{\text {a }}$ \\
\hline N04B D Selegilina 54,4 (28,7) & 6,5 & 19,4 & 31,3 & 39,8 & 57,7 & 68,7 & 78,7 & 82,9 & 82,4 & 98,0 & 1269 \\
\hline N04B X Tolcapona & & & & & & & & &
\end{tabular}

Tolcapona

7,2

X (DE) anual: media de consumo anual durante todo el período de estudio. \% var.: porcentaje de variación [(consumo 1998 - consumo 1989)/consumo 1989]× 100. a Porcentaje de variación [(consumo 1998 - consumo 1991)/consumo 1991] × 100. ${ }^{\mathrm{b}}$ Porcentaje de variación [(consumo 1998 - consumo 1997)/consumo 1997] $\times 100$.

los resultados indican que también es el más consumido (alrededor de un $40 \%$ del consumo total). El consumo de levodopa es un buen indicador de la prevalencia de la EP, ya que su única indicación es el tratamiento de dicha enfermedad. Diversos estudios han estimado la prevalencia a de la EP a partir de los datos de consumo de levodopa [11,14,17-21,24-26]. El consumo de levodopa en nuestro estudio ha sido superior al observado en otro estudio de ámbito nacional (0,50 DDD/1.000 habitantes/día), pero con datos de un período previo (1982-1984) [10]. Los estudios realizados en distintas comunidades autónomas también muestran que la levodopa es el fármaco antiparkinsoniano más consumido, aunque el consumo es muy variable (desde 0,6 hasta 2,7 DDD/1.000 habitantes/día) [11-13,15]. En este sentido, un estudio de ámbito nacional que sólo analizó el consumo de levodopa durante el período 1990-1995 mostró una gran variabilidad geográfica, con un mayor consumo en las provincias del norte que en las del sur de España [15]. Además, también se ha señalado que el consumo de levodopa en España es inferior al de los países nórdicos [14].

Un hallazgo muy significativo del estudio es la evolución del consumo de selegilina, ya que desde su comercialización ha sido el fármaco que ha experimentado un mayor crecimiento, y es el segundo fármaco más consumido. Nuestros resultados, de ámbito nacional, son similares a los de otros estudios de ámbito local, que también han constatado un aumento de consumo de la selegilina, y han indicado que es el segundo fármaco más consumido tras la levodopa [11-13]. Por otra parte, los datos de consumo de selegilina apuntan que la publicación del ensayo clínico PDRGUK [7] ha tenido un reducido impacto sobre el consumo (Fig. 2). La publicación de este ensayo clínico [7], en 1995, y un reanálisis posterior [27], en 1998, planteó serias dudas sobre la seguridad de la selegilina, porque constató un aumento de la mortalidad en los pacientes tratados con dicho medicamento. Este estudio generó una intensa polémica, y se argumentó que existían limitaciones metodológicas que podían sesgar los resultados, porque no se realizó un enmascaramiento del tratamiento; tampoco fue posible el seguimiento de un porcentaje elevado de pacientes, ni se dispuso de información muy detallada sobre las causas de la muerte [28,29]. Durante los años siguientes a la publicación del estudio, la controversia ha persistido, porque los resultados de estudios de observación han sugerido un pequeño aumento de la mortalidad asociado al tratamiento con selegilina [30]; pero, en cambio, los resultados de un metanálisis [31] y de estudios de observación [32,33] han concluido que no existía un incremento de la mortalidad relacionado con el uso de selegilina.

El consumo global de los agonistas dopaminérgicos también se ha incrementado, aunque se observan algunas diferencias entre los fármacos de este grupo. El consumo de pergolida y ropinirol aumenta, el de lisurida se mantiene y el de bromocriptina disminuye. Interesa destacar que los fármacos de este grupo, cuya única indicación aprobada es la EP, fueron los que 
Tabla IV. Evolución anual del consumo de los nuevos fármacos.

\begin{tabular}{|c|c|c|c|c|}
\hline Año & $\begin{array}{c}\text { Consumo } \\
\text { nuevos fármacos }\end{array}$ & $\begin{array}{l}\text { Consumo } \\
\text { fármacos clásicos }\end{array}$ & $\begin{array}{l}\text { Consumo nuevos } \\
\text { fámmacos }(\%)^{b}\end{array}$ & $\begin{array}{l}\text { Consumo fármacos } \\
\text { clásicos }(\%)^{\mathrm{b}}\end{array}$ \\
\hline 1990 & 0,001 & 2,11 & 0,05 & 99,95 \\
\hline 1991 & 0,03 & 2,34 & 1,3 & 98,7 \\
\hline 1992 & 0,07 & 2,48 & 2,7 & 97,3 \\
\hline 1993 & 0,08 & 2,70 & 2,9 & 97,1 \\
\hline 1994 & 0,09 & 2,86 & 3,0 & 97,0 \\
\hline 1995 & 0,12 & 3,01 & 3,2 & 96,8 \\
\hline 1996 & 0,12 & 3,26 & 3,5 & 96,5 \\
\hline 1997 & 0,13 & 3,24 & 4,0 & 96,0 \\
\hline 1998 & 0,24 & 3,40 & 6,6 & 93,4 \\
\hline Media (DE) & $0,09(0,06)$ & $2,82(2,1)$ & $2,7(1,2)$ & $96,9(1,8)$ \\
\hline
\end{tabular}

${ }^{a}$ Consumo expresado en DDD/1.000 habitantes/día. ${ }^{b}$ Porcentaje del consumo total de fármacos antiparkinsonianos.

Tabla V. Evolución anual del gasto monetario de los nuevos fármacos.

\begin{tabular}{|c|c|c|c|c|}
\hline Año & $\begin{array}{l}\text { Gasto nuevos } \\
\text { fármacos }(\%)^{\mathrm{a}}\end{array}$ & $\begin{array}{l}\text { Gasto fármacos } \\
\text { clásicos }(\%)^{\mathrm{a}}\end{array}$ & $\begin{array}{c}\text { Coste DDD } \\
\text { nuevos fámacos }\end{array}$ & $\begin{array}{l}\text { Coste DDD } \\
\text { fármacos clásicos }\end{array}$ \\
\hline 1990 & 0,2 & 99,8 & 1,86 & 0,53 \\
\hline 1991 & 6,7 & 93,3 & 2,55 & 0,54 \\
\hline 1992 & 12,6 & 87,4 & 2,89 & 0,58 \\
\hline 1993 & 13,7 & 86,3 & 2,88 & 0,59 \\
\hline 1994 & 14,3 & 85,7 & 2,87 & 0,60 \\
\hline 1995 & 15,2 & 84,8 & 3,12 & 0,62 \\
\hline 1996 & 16,8 & 83,2 & 3,41 & 0,63 \\
\hline 1997 & 20,5 & 79,5 & 3,88 & 0,64 \\
\hline 1998 & 38,8 & 61,1 & 13,59 & 0,62 \\
\hline Media (DE) & 15,4 (11) & 84,6 (11) & $4,13(3,59)$ & $0,59(0,04)$ \\
\hline
\end{tabular}

a Porcentaje del coste total de todos los fármacos antiparkinsonianos. ${ }^{b}$ Coste de una DDD expresado en $j$.

agonistas dopaminérgicos ha generado gran interés, como fármacos coadyuvantes o alternativos a la levodopa [34,35]. Aunque se acepta la utilización de estos nuevos agonistas dopaminérgicos como tratamiento adicional en las fases avanzadas de la EP, su papel en las fases iniciales de la enfermedad es controvertido. Los resultados de ensayos clínicos recientes [36,37], que han comparado el tratamiento inicial con levodopa o los nuevos agonistas dopaminérgicos (ropirinol, pramipexol), indican que la levodopa es más eficaz en la mejoría de las alteraciones motoras de los pacientes, si bien los nuevos agonistas dopaminérgicos se asocian a menos efectos adversos motores, sobre todo discinesias. Sin embargo, el tratamiento con los nuevos agonistas dopaminérgicos se relaciona con mayor frecuencia a otros efectos indeseados, como, por ejemplo, alucinaciones, somnolencia y edema [36-38]. Además, también se han descrito ataques de sueño súbito asociados al uso de los nuevos agonistas dopaminérgicos no ergóticos, que en algunos casos han causado accidentes de tráfico [39-42]. El consumo de anticolinérgicos prácticamente no se ha incrementado durante todo el período de estudio, y el de amantadina es muy escaso y ha disminuido. Estos datos sugieren que son fármacos en desuso, ya que existen otras alternativas farmacológicas con una mejor relación beneficio/riesgo, y que el crecimiento del consumo se desplaza hacia otros fármacos. No obstante, pueden existir diferencias geográficas en el consumo, tanto de los anticolinérgicos como de amantadina, porque se observan diferencias en los datos de consumo entre diferentes estudios de ámbito local [11-13].

El impacto de los nuevos fármacos antiparkinsonianos comercializados durante la década de los años noventa, medi-

aumentaron más su consumo, porque algunos agonistas dopaminérgicos también se utilizan en el tratamiento de otras enfermedades (bromocriptina, lisurida y cabergolina). El consumo de bromocriptina y el de lisurida fueron similares a los observados en los estudios de consumo realizados en las comunidades de Navarra [11] y Castilla-La Mancha [13]. En cambio, el consumo de pergolida en Castilla-La Mancha [13] fue unas 10 veces superior al observado en Navarra [11] y al de la media nacional observado en nuestro estudio. Las diferencias geográficas en los hábitos de prescripción podrían explicar estas diferencias geográficas de consumo, pero, en nuestro estudio de ámbito nacional, no se analizaron. Un resultado novedoso de nuestro estudio fue la evaluación del consumo de ropirinol, que no se contempló en estudios anteriores. Sin embargo, no se pudo analizar el consumo de pramipexol, ya que se comercializó en diciembre de 1998. La comercialización de estos nuevos do en unidades de consumo, ha sido modesto. Sin embargo, el impacto económico ha sido muy grande, porque se ha incrementado el gasto desde un $7 \%$ del gasto total en 1991, hasta un $39 \%$ en 1998, como consecuencia de su elevado precio. Cabe destacar el fuerte impacto que han tenido los inhibidores de COMT sobre el consumo, tanto en términos de unidades de consumo como monetarias. El consumo de la tolcapona -el primer medicamento comercializado de este grupo-durante el primer año de su comercialización fue tan importante que incluso superó al consumo de bromocriptina, lisurida y ropirinol. Sin embargo, la tolcapona se retiró del mercado de la Unión Europea y del canadiense porque se observaron varios casos de hepatitis entre los pacientes tratados, y, además, algunos fueron hepatitis fulminantes $[8,43]$. Por otra parte, la comercialización de entacapona, otro inhibidor de la COMT, fue posterior al período de estudio, y, obviamente, no pudo analizarse su consu- 


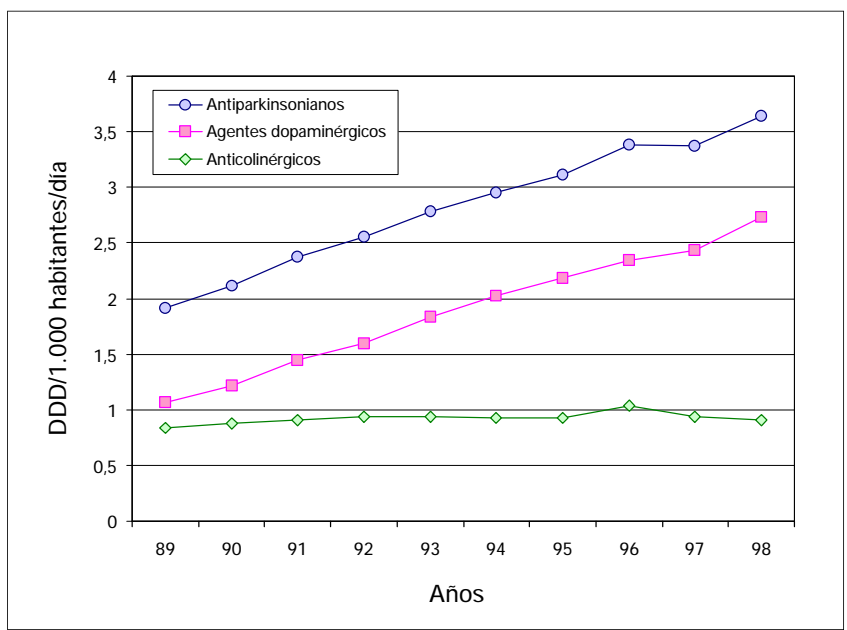

Figura 1. Evolución anual del consumo de fármacos antiparkinsonianos.

mo. Las principales limitaciones de nuestro estudio se relacionan con la fuente de identificación de los datos, la base ECOM, del Ministerio de Sanidad y Consumo, que sólo incluye la información sobre el consumo de especialidades dispensadas en oficinas de farmacia con cargo a la Seguridad Social. Esta base de datos no incluye prescripciones realizadas para los beneficiarios de otras entidades aseguradoras como MUFACE o ISFAS, ni tampoco los datos de venta directa; probablemente, este consumo representa una reducida proporción del consumo total. Por otra parte, algunos de los fármacos analizados también se utilizan en otras indicaciones diferentes de la EP (anticolinérgicos, bromocriptina, cabergolina, etc.); no obstante, el consumo total de estos fármacos se ha reducido. También se debe destacar que los datos analizados corresponden a las ventas de medicamentos, y no todos los fármacos vendidos los consumen los pacientes. Finalmente, en nuestro estudio no se han podido analizar los datos de consumo de algunos de los nuevos fármacos (pramipexol y en-

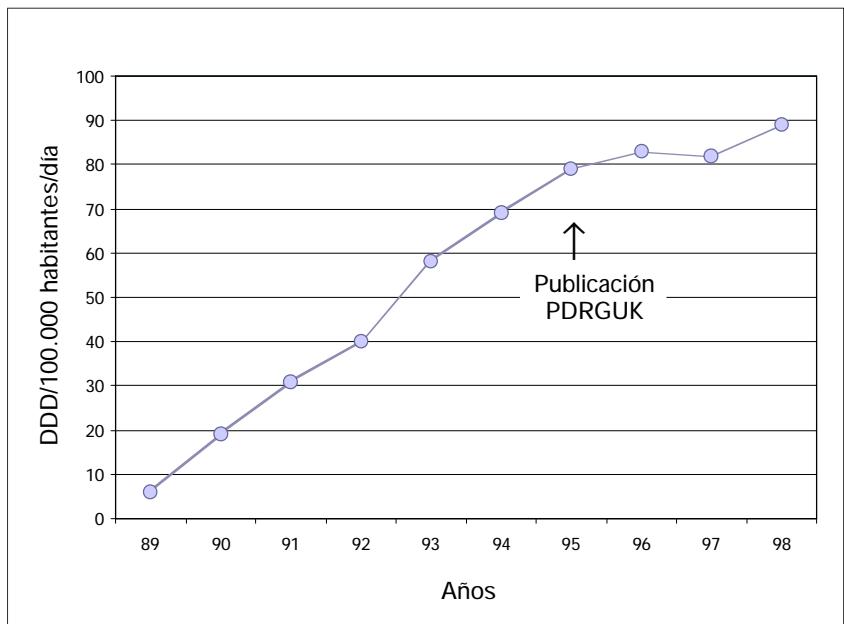

Figura 2. Impacto del estudio PDRGUK (Parkinson's Disease Research Group of the United Kingdom) [7] en el consumo anual de selegilina.

tacapona), porque su comercialización fue posterior a la del período estudiado, pero estas limitaciones no invalidan los resultados del estudio, que identifica un aumento del consumo de los fármacos antiparkinsonianos de ámbito nacional y cambios en el patrón de consumo a lo largo del tiempo. Además, los resultados de nuestro estudio tienen interés porque se han analizado algunos fármacos de reciente comercialización, de los que no existían datos de consumo. Se necesitarán futuros estudios para conocer la evolución a largo plazo de estos fármacos y analizar el consumo de los nuevos fármacos ya comercializados, pero que no se han evaluado en nuestro estudio.

En resumen, el consumo de fármacos antiparkinsonianos ha aumentado durante la última década, sobre todo a expensas del consumo de levodopa, de selegilina y de los agonistas dopaminérgicos. Los nuevos fármacos antiparkinsonianos han tenido un gran impacto económico. La polémica sobre la seguridad de la selegilina ha tenido un escaso impacto sobre su consumo.

\section{BIBLIOGRAFÍA}

1. Lang AE, Lozano AM. Parkinson's Disease. N Engl J Med 1998; 339: 1044-53.

2. Young R. Update on Parkinson's Disease. Am Fam Phisician 1999, 59; 2155-67.

3. Jiménez-Jiménez FJ. Tratamiento de la enfermedad de Parkinson. Med Clin (Barc) 1999; 112: 257-8.

4. Schapira AHV. Parkinson's disease. Br Med J 1999; 318: 311-4.

5. Cummings JL. Understanding Parkinson Disease. JAMA 1999; 281: 376-8.

6. De Rijk MC, Tzourio C, Breteler MM, Dartigues JF, Amuducci L, López-Pousa, et al. Prevalence of parkinsonism and Parkinson's disease in Europe: the EUROPARKINSON Collaborative Study. European Community Concerted Action on the Epidemiology of Parkinson's disease. J Neurol Neurosurg Psychiatry 1997; 62: 10-5.

7. Lees AJ. On behalf of the Parkinson's Disease Research Group of the United Kingdom. Comparison of therapeutic effects and mortality of levodopa and levodopa combined with selegiline in patients with early, mild Parkinson's disease. Br Med J 1995; 311: 1602-7.

8. Kaakola S. Clinical pharmacology, therapeutic use and potential of COMT inhibitors in Parkinson disease. Drugs 2000; 59: 1233-50.

9. Olanow CW. Tolcapone and hepatotoxicity. Tasmar Advisory Panel. Arch Neurol 2000; 57: 263-7.

10. Limón-Mendizábal C, García-Iñesta A, Ortega-Limón A. Utilización de levodopa en España en el período 1982-84. Inf Ter Segur Soc 1985; 9: 202-10.

11. Artazcoz MT, Viñes JJ. Estimación de la prevalencia de la enfermedad de Parkinson en Navarra. Estudio farmacoepidemiológico del consumo de antiparkinsonianos. Rev Esp Salud Pública 1995; 69: 479-85.

12. Manzanares R, Matías-Guiu J, Provenció R, Falip R, López-Arlandis J, Martín R, et al. Dosis diarias definidas de fármacos antiparkinsonianos en Alcoi. Rev Neurol 1996; 24: 440-2.
13. Criado-Álvarez JJ, Romo-Barrientos C, Martínez-Hernández J, González-Solana I. Consumo de antiparkinsonianos en Castilla-La Mancha. Estimación de la prevalencia de la enfermedad de Parkinson. Rev Neurol 1998; 27: 405-8.

14. Cuadrado JI, Pedro-Cuesta J, Abraira V, Stawiarz L, Iñesta A, Almazán J. Epidemiological assessment of levodopa use in Spain, 1990-1995. Persistent, low consumption in the south. Pharmacoepidemiol Drug Saf 1999; 8: 433-45.

15. Martínez-Suárez MM, Blázquez-Menes B. Estimación de la prevalencia de la enfermedad de Parkinson en Asturias, España. Un estudio farmacoepidemiológico del consumo de fármacos antiparkinsonianos. Rev Neurol 2000; 31: 1001-6.

16. Anónimo. Guidelines for ATC classification and DDD assignment. Oslo: WHO Collaborating Centre for Drug Statistics Methodology; 1998.

17. De Pedro J, Rosenqvist U. Tracers for paralysis agitants in epidemiological research. III. Refinement of the model for estimation of the prevalence of the disease. Neuroepidemiology 1985; 4: 161-75.

18. De Pedro J, Rosenqvist U. Tracers for paralysis agitants in epidemiological research. IV. Trends in national drug policy and measurement of the prevalence of the disease in Sweden. Neuroepidemiology 1985; 4: 161-75.

19. De Pedro-Cuesta J, Petersen IJ, Vassilopoulos D, Micheli F, GarcíaIniesta A. Epidemiological assessment of levodopa use by populations. Acta Neurol Scand 1991: 83: 328-35.

20. De Pedro-Cuesta J, Petersen IJ, Stawiarz L, Gudmundsson G, Almazán J, Tulinius $\mathrm{H}$, et al. High levodopa use in periodically time-clustered, Icelandic birth cohorts. A vestige of parkinsonism etiology? Europarkinson Preparatory Activity Research Group. Acta Neurol Scand 1995; 91: 79-88.

21. De Pedro-Cuesta J, Wermuth L, Abraira V, Stawiarz L. Levodopa use in Denmark: high levels in Grenland and the Faroe Islands. Europar- 
kinson Preparatory Activity Research Group. Acta Neurol Scand 1995; 91: 89-97.

22. Sevillano-García MD, Cuadrado-Gamarra JI, de Pedro-Cuesta J. Enfermedad de Parkinson en España: evidencias de infradiagnóstico y puntos de partida para su reducción. Rev Neurol 1999; 29: 881-3.

23. Errea JM, Ara JR, Aibar C, de Pedro-Cuesta J. Prevalence of Parkinson's disease in lower Aragon, Spain. Mov Disord 1999; 14: 596-604.

24. Kleinhenz J, Vieregge P, Fassl H, Jorg J. The prevalence of Parkinson disease in West Germany are general practice data a suitable survey instrument? Offentl Gesundheitswess 1990; 52: 181-90.

25. Menniti-Ippolito F, Spila-Alegiani S, Vanacore N, Bonifati V, Diana G, Meco G, et al. Estimate of parkinsonism prevalence through drug prescription histories in the Province of Rome, Italy. Acta Neurol Scand 1995; 92: 49-54.

26. Lanska DJ. Comparison of utilization of Sinemet and Parkinson's disease mortality as surrogate indicators of Parkinson's disease in the United States. J Neurol Sci 1997; 145: 105-8.

27. Ben-Sholomo Y, Churchyard A, Head J, Hurwitz B, Overstall P, Ockelford J, et al. Investigation by Parkinson's Disease Research Group of United Kingdom into excess mortality seen with combined levodopa and selegiline treatment in patients with early, mild Parkinson's disease: further results of randomised trial and confidential inquiry. Br Med J 1998; 316: 1191-6.

28. Heinonen EH, Myllylä V. Safety of selegiline (deprenyl) in the treatment of Parkinson's disease. Drug Safety 1998; 19: 11-22.

29. Breteler MMB. Selegiline, or the problem of early termination of clinical trials. The clinical questions are not well answered, and probably never will be. Br Med J 1998; 316: 1182-3.

30. Thorogood M, Amstrong B, Nichols T, Hollowell J. Mortality in people taking selegiline: observational study. Br Med J 1998; 317: 252-4.

31. Olanow CW, Myllyla VV, Sotaniemi KA, Larsen JP, Palhagen S, Przuntek H, et al. Effect of selegiline on mortality in patients with Parkinson's disease: a meta-analysis. Neurology 1998; 51: 825-30.

\section{EVOLUCIÓN DEL CONSUMO DE FÁRMACOS ANTIPARKINSONIANOS EN ESPAÑA}

Resumen. Introducción. En los últimos años se han comercializado nuevos fármacos antiparkinsonianos y ha existido una polémica sobre la seguridad de algunos de ellos. Objetivo. Analizar la evolución del consumo de los fármacos antiparkinsonianos y el impacto de los nuevos fármacos. Material y métodos. Estudio de consumo de los fármacos antiparkinsonianos (1989-1998). Los datos se obtuvieron de las bases ECOM, del Ministerio de Sanidady Consumo, y TEMPUS, del Instituto Nacional de Estadística. Los fármacos se clasificaron mediante la Clasificación Anatómica-TerapéuticaQuímica (ATC). El consumo se expresó en dosis diarias definidas (DDD), y los costes, en euros ( $n$ ). Los fármacos comercializados desde 1990 se clasificaron como nuevos fármacos, y el resto, como fármacos clásicos. Resultados. El consumo total de los fármacos aumentó desde 1,92 DDD/1.000 habitantes/día, en 1989, hasta 3,64 DDD/1.000 hab./día, en 1998. Los fármacos con un mayor incremento del consumo fueron selegilina, pergolida y levodopa. El coste farmacéutico total se triplicó. El aumento del consumo de los nuevos fármacosfue menor (1,2\% del total en 1991 y 6,6\% en 1998) que el de sus costes (6,7\% del total en 1991 y 38,8\% en 1998). El coste por DDD de los nuevos fármacos aumentó cinco veces (1989: 2,55n y 1998: 13,59n y el de los fármacos clásicos fue similar (1989: 0,54n y 1998: 0,62n). Conclusiones. El consumo total de fármacos antiparkinsonianos aumenta progresivamente. El consumo de selegilina también ha aumentado, a pesar de la polémica sobre su seguridad. Los nuevos fármacos tienen un gran impacto económico. [REV NEUROL 2002; 34: 612-7]

Palabras clave. Agentes antiparkinsonianos. Análisis de costes. Costes. Costes farmacológicos. Enfermedad de Parkinson. Terapia farmacológica. Utilización de fármacos.
32. Parkinson Study Group. Mortality in DATATOP: a multicenter trial in early Parkinson's disease. Parkinson Study Group. Ann Neurol 1998; 43: 318-25.

33. Donnan PT, Steinke DT, Stubbings C, Davey PG, MacDonald TM. Selegiline and mortality in subjects with Parkinson's disease: a longitudinal community study. Neurology 2000; 55: 1785-9.

34. Adler C, Sethi K, Hauser R, Davis T, Hammersted J, Bertoni J, et al. Ropirinole for the treatment of early Parkinson's disease. Neurology 1997; 9: 393-9.

35. Shannon K, Bennett J, Friedman J, for the Parkinson Study Group. Efficacy of pramipexole, a novel dopamine agonist, as monotherapy in mild to moderate Parkinson's disease. Neurology 1997; 49: 724-8.

36. Parkinson Study Group. Pramipexole vs levodopa as initial treatment for Parkinson disease: a randomized controlled trial. JAMA 2000; 284: 1931-8.

37. Rascol O, Brooks D, Korczyn A, De Deyn P, Clarke C, Lang A, et al. for the 056 Study Group. A five-year study of the incidence of dyskinesia in patients with early Parkinson's disease who were treated with ropirinole or levodopa. N Engl J Med 2000; 342: 1484-91.

38. Tanner CM. Dopamine agonists in early therapy for Parkinson disease. Promise and problems. JAMA 2000; 284: 1971-3.

39. Frucht S, Rogers JD, Greene PE, Gordon MF, Fahn S. Falling asleep at the wheel: motor vehicle mishaps in persons taking pramipexole and ropirinole. Neurology 1999; 52: 1908-10.

40. Olanow CW, Schapira AH, Roth T. Waking up to sleep episodes in Parkinson's diseases. Mov Disord 2000; 15: 212-5.

41. Ryan M, Slevin JT, Wells A. Non-ergot dopamine agonist-induced sleep attacks. Pharmacother 2000; 20: 724-6.

42. Anónimo. Antiparkinsonian drugs and 'sleep attacks'. Can Adverse Drug React News 2001; 164: 1038-9.

43. Assal F, Spahr L, Hadengue A, Rubbia-Brandt L, Burkhard PR. Tolcapone and fulminant hepatitis. Lancet 1998; 352: 958.

\section{EVOLUÇÃO DO CONSUMO DE FÁRMACOS ANTIPARKINSÓNICOS EM ESPANHA}

Resumo. Introdução. Nos últimos anos foram lançados no mercado novos antiparkinsónicos, tendo-se levantado uma polémica sobre a segurança de alguns fármacos. Objectivo. Analisar a evolução do consumo dos antiparkinsónicos e novos fármacos. Material e métodos. Etudio do consumo dos antiparkinsónicos (1989-1998). Os dados foram obtidos das bases ECOM, do Ministério da Saúde e Consumo, e TEMPUS, do Instituto Nacional de Estatística. Os fármacos foram classificados pela Classificação Anatómico-Terapêutico-Química (ATC). O consumo expressouse em dose diária definida (DDD), e os custos em euros (n). Os fármacos comercializados desde 1990 foram classificados como novos fármacos e os restantes como fármacos clássicos. Resultados. O consumo total dos fármacos aumentou desde 1,92 DDD/ 1.000 habitantes/dia em 1989 até 3,64 DDD/1.000 hab./dia em 1998. Os fármacos com maior incremento de consumo foram a selegilina, pergolida e levodopa. O custo farmacêutico total triplicou. $O$ aumento do consumo dos novos fármacos foi menor (1,2\% do total em 1991 e 6,6\% em 1998) do que os seus custos (6,7\% do total em 1991 e 38,8\% em 1998). O custo por DDD dos novos fármacos aumentou cinco vezes (1989: 2,55n e 1998: 13,59n) e o dos fármacos clássicos foi similar (1989: 0,54n e 1998: 0,62n. Conclusões. $O$ consumo total de fármacos antiparkinsónicos aumentou progressivamente. $O$ consumo de selegilina também aumentou apesar da polémica sobre a sua segurança. Os novos fármacos tiveram um grande impacto económico. [REV NEUROL 2002; 34: 612-7]

Palavras chave. Agentes antiparkinsónicos. Análise de custos. Custos. Custos farmacológicos. Doença de Parkinson. Terapia farmacológica. Utilização de fármacos. 